\title{
Topological origin of subgap conductance in insulating bilayer graphene
}

\author{
Jian $\mathrm{Li}^{1}$, Ivar Martin ${ }^{2}$, Markus Büttiker' and Alberto F. Morpurgo ${ }^{3 \star}$
}

\begin{abstract}
The edges of graphene-based systems possess unusual electronic properties, originating from the non-trivial topological structure associated with the pseudospinorial character of the electron wavefunctions. These properties, which have no analogue for electrons described by the Schrödinger equation in conventional systems, have led to the prediction of many striking phenomena, such as gate-tunable ferromagnetism and valley-selective transport ${ }^{1-3}$. In most cases, however, the predicted phenomena are not expected to survive the strong structural and chemical disorder that unavoidably affects the edges of real graphene devices. Here, we present a theoretical investigation of the intrinsic low-energy states at the edges of electrostatically gapped bilayer graphene, and find that the contribution of edge modes to the linear conductance of realistic devices remains sizable even for highly imperfect edges. This contribution may dominate over that of the bulk for sufficiently clean devices, such as those based on suspended bilayer graphene samples. Our results illustrate the robustness of those phenomena whose origin is rooted in the topology of the electronic band structure, even in the absence of specific protection mechanisms.
\end{abstract}

The electronic structure of graphene single and bilayers possesses non-trivial topological properties, which originate from the spinorial nature of the electronic wavefunctions, and which manifest themselves in many ways. For instance, when transported along a closed trajectory, the wavefunction of an electron in single- or bilayer graphene acquires a non-vanishing Berry phase, responsible for the specific quantization sequence of the Hall conductance that is observed experimentally ${ }^{4-6}$. Another manifestation of the topological properties is the presence of zeroenergy states living at the material edges ${ }^{7-9}$. The existence of these states is enabled by the richer structure of edge boundary conditions (as compared with the conventional scalar wavefunctions) that is associated with the spinorial nature of the electronic wavefunctions (see, for example, ref. 10).

Many fascinating phenomena originating from the properties of edge states in graphene layers have been predicted theoretically, such as electric-field-tunable magnetism ${ }^{1}$ and valley-dependent transport ${ }^{2,3}$. In most cases, however, the possibility of observing these phenomena is dubious, because theory often relies on a perfectly ordered atomic structure of the graphene edges, whereas in real materials strong disorder is unavoidably present. To examine whether exotic aspects of the edge physics can survive disorder, we analyse the transport properties of low-energy edge states in the case of gapped bilayer graphene (BLG) (ref. 11). Contrary to the naive expectation, we show that transport mediated by the low-energy edge states exhibits universal features, and persists even in the presence of very strong disorder.
To illustrate the topological origin of edge states in gapped BLG (Fig. 1a), we describe the low-energy electronic properties of this material in terms of an effective (dimensionless) Hamiltonian, by now well established both theoretically and experimentally ${ }^{11}$ :

$$
\begin{gathered}
H_{\tau}=-\mathbf{g}_{\tau}\left(k_{x}, k_{y}\right) \cdot \boldsymbol{\sigma} \\
\mathbf{g}_{\tau}\left(k_{x}, k_{y}\right)=\left(k_{x}^{2}-k_{y}^{2}, 2 k_{x} k_{y} \tau, \Delta\right)
\end{gathered}
$$

where $\boldsymbol{\sigma}$ stands for the vector of Pauli matrices, $\tau= \pm 1$ indexes the two valleys in the band structure (corresponding to the $\mathrm{K}$ and $\mathrm{K}^{\prime}$ points in the Brillouin zone), $\mathbf{k}=\left(k_{x}, k_{y}\right)$ is the momentum relative to the $\mathrm{K} / \mathrm{K}^{\prime}$ point and $2 \Delta$ defines the size of the gap. This Hamiltonian - which can be derived as a long-wavelength low-energy limit of a tight-binding description-acts on spinors, whose components correspond approximately to the amplitude of the wavefunction on atoms A1 and B2 of the bilayer unit cell (see Supplementary Information for details). The non-trivial topological properties of BLG are described by the integer ${ }^{12,13}$

$$
c_{\tau}=\frac{1}{4 \pi} \int \mathrm{d} k_{x} \int \mathrm{d} k_{y} \hat{\mathbf{g}}_{\tau} \cdot\left(\frac{\partial \hat{\mathbf{g}}_{\tau}}{\partial k_{x}} \times \frac{\partial \hat{\mathbf{g}}_{\tau}}{\partial k_{y}}\right)
$$

associated with the mapping from the region of the Brillouin zone that contains the valley point $\mathrm{K} / \mathrm{K}^{\prime}$ to the unit vector $\boldsymbol{g}_{\tau}$ defining the single-valley Hamiltonian $\left(c_{\tau}\right.$ is equal to 1 in one of the valleys and -1 in the other). By analogy with the bulk-edge correspondence $^{14,15}$, it can then be expected that zero-energy states, propagating in opposite directions in the two valleys, appear at the material edge as a consequence of the discontinuity of the topological charge between BLG and the vacuum. Indeed, 'valleyhelical' states appear at interfaces separating regions of BLG where the gaps have opposite polarities ${ }^{13}$, and have been shown to exist at zigzag edges in ref. 8 (see also Supplementary Information). In a device consisting of a strip of BLG with zigzag edges, these zeroenergy states propagate ballistically and are responsible for a finite conductance equal to $4 e^{2} / h$ (corresponding to two spin-degenerate states at each one of the device edges) when the Fermi level is located inside the gap.

These properties of BLG parallel those of spin-orbit induced topological insulators ${ }^{15-18}$, in which ballistic helical edge states have been observed experimentally ${ }^{19,20}$, but with the valley quantum number playing the role of the electron spin. A complete analogy between BLG and spin-orbit induced topological insulators, however, is prevented by a number of important differences that will be discussed in detail elsewhere. It is crucial in the present case that edge states in spin-orbit-induced topological insulators cannot back-scatter (and hence do not localize) as long as time-reversal

'Département de Physique Théorique, Université de Genève, CH-1211 Genève 4, Switzerland, ${ }^{2}$ Theoretical Division, Los Alamos National Laboratory, Los Alamos, New Mexico 87545, USA, ${ }^{3}$ DPMC and GAP, Université de Genève, CH-1211 Genève 4, Switzerland. *e-mail: alberto.morpurgo@unige.ch. 

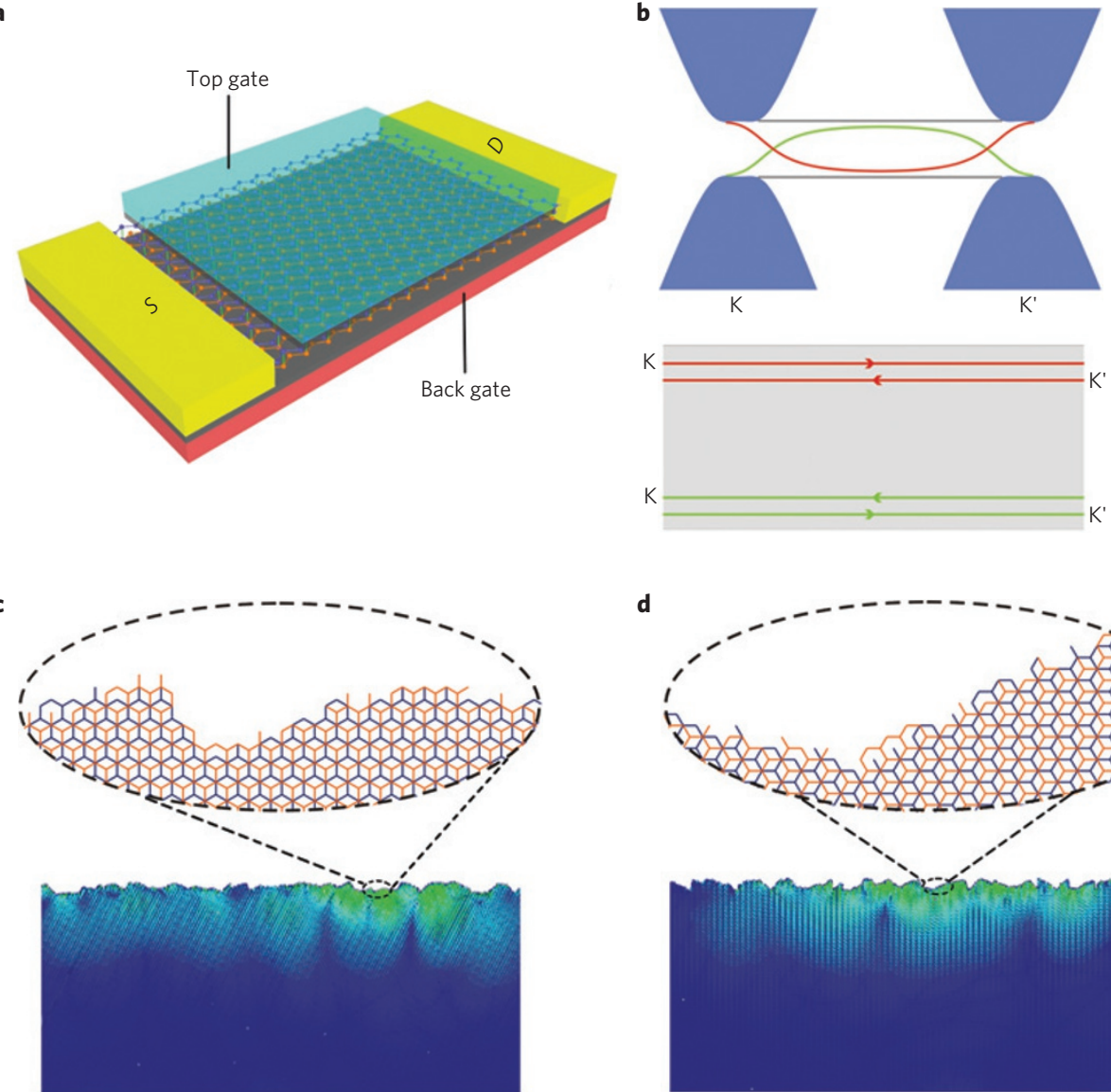

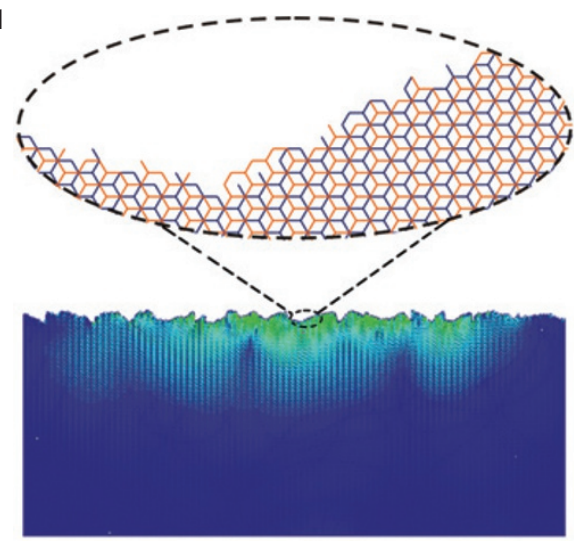

Figure 1 | Edge states in gapped BLG. a, A schematic view of the device. By controlling the gate voltages applied to the top and back gates separately, an energy gap in the bulk of BLG can be opened and tuned, while maintaining the Fermi energy in its centre. $\mathbf{b}$, The dispersion of the subgap edge states in gapped BLG at a zigzag edge. At both edges, the states are helical with respect to the valley degree of freedom, with states in opposite valleys propagating in opposite directions. This conclusion holds for periodic edges of rather general shape (that is, not only for zigzag), but it does not hold for the ideal armchair edge, where two valleys ( $K$ and $K^{\prime}$ ) are fully coupled and no subgap edge states are present. c,d, The typical probability density near zero energy for strongly disordered BLG zigzag (c) or armchair (d) edges. In the presence of realistically strong disorder, the existence of such edge states-and their long localization length-is a universal property of gapped BLG (the panels correspond to BLG that is approximately 100 nm long).

symmetry is present-a phenomenon referred to as topological protection $^{16-18}$. In graphene bilayers, on the other hand, any mechanism coupling states in different valleys can affect the edge states. For this reason, it might be expected that any source of short-range scattering due to the edge non-ideality would make the behaviour found for ideal zigzag edges irrelevant for the description of transport in real devices.

To analyse the properties of the low-energy states present at disordered edges we go back to the tight-binding description of gapped BLG, and carry out numerical calculations of conductance for strips of gapped BLG. Short-range disorder is introduced in two different ways, which model different physical phenomena occurring at real graphene edges. Structural edge disorder is generated by randomly removing tight-binding sites in the two layers ${ }^{21}$, up to a depth $d$ from the starting edge (see examples in Fig. 1c,d). The effect of generic chemical species binding to the outermost carbon atoms is modelled by adding to the corresponding sites in the tight-binding Hamiltonian an energy that is distributed randomly in the range $\pm 1 \mathrm{eV}$ (that is, the characteristic order of magnitude associated with chemical bonds). We carry out calculations starting from different 'ideal' edges, including zigzag, armchair and other edges generated by cutting the BLG along arbitrary crystallographic directions, for many different microscopic realizations of disorder, from which we extract the ensemble-averaged conductance. In the calculations, we use the known values for the in- and out-of-plane hopping parameters of BLG, and gap values in the range that is accessible to experiments (see Methods Section).

The ensemble-averaged conductance calculated as a function of sample length $L$ for different values of $d$ is shown in Fig. 2a,b, corresponding to the cases of starting ideal zigzag and armchair edges, respectively. It is apparent that in all cases the average conductance decays exponentially with $L$. The calculated conductance is independent of the width $W$ of the graphene strip, indicating that transport is indeed occurring along the edges (see Fig. 2d). Only for very short strips $(L<5 \mathrm{~nm})$ is a sizable 'bulk' contribution to the conductance (scaling linearly with the width of the strip) also significant, owing to direct tunnelling under the gap. This contribution decays very rapidly on increasing the device length, and can be ignored in the range of values of $L$ that we are considering. Note also that the conductance calculated for large values of $L$ extrapolates in all cases to $4 e^{2} / h$ for $L \rightarrow 0$, as expected for edge transport (the factor of four accounts for two edges and two spin directions). Therefore, the observed exponential decay of conductance $G$ as a function of $L$ enables us to directly extract the localization length $\lambda_{\text {loc }}$ of the edge states.

When starting from an ideal zigzag edge, structural disorder causes localization of the pre-existing zero-energy states, and the observed decrease of $\lambda_{\text {loc }}$ with increasing $d$ is expected (see Fig. 3; the case where only structural disorder is considered corresponds to the points connected by the continuous line). Unexpectedly, 


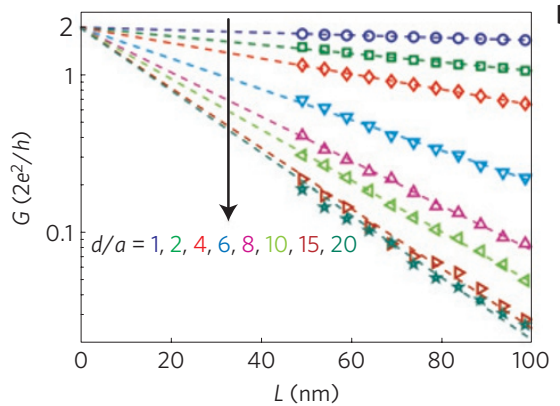

c

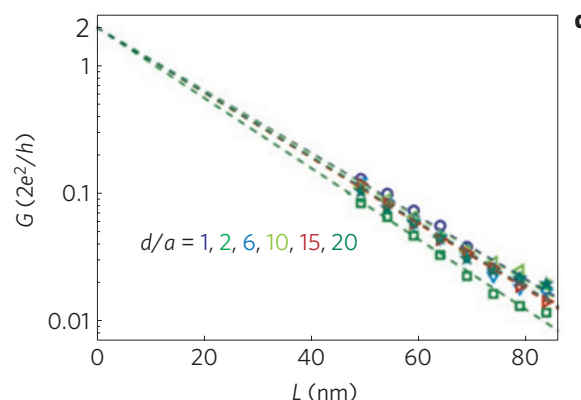

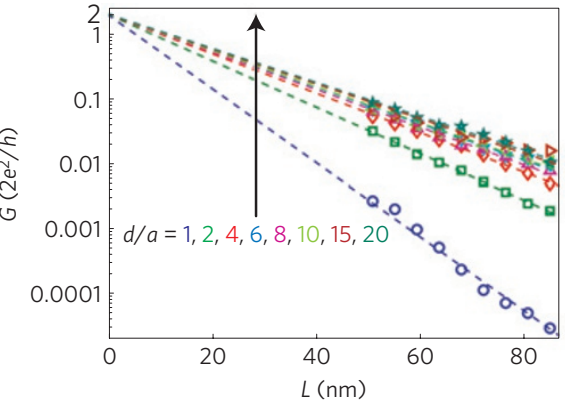

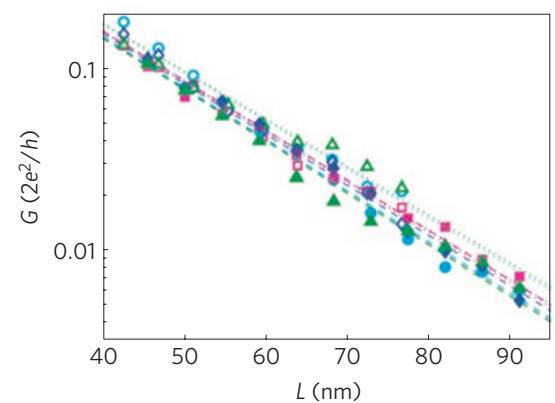

Figure 2 | Subgap conductance in disordered BLG. a-d, The decay of average $G$ (at $E_{f}=0$ ) with increasing length $L$ of the BLG strips, for different amounts of disorder. $\mathbf{a}, \mathbf{b}$, The arrows indicate that the evolution of the conductance with increasing structural edge roughness (quantified by the edge roughness depth $d$ ) is opposite for samples starting from ideal zigzag (a) and armchair (b) edges. The functional dependencies of the conductance on $L$ calculated for different values of $d$ converge to the same universal limit when disorder is sufficiently strong (that is, the $G(L)$ traces become universal at large $d$ ). $\mathbf{c}$, In the presence of 'chemical' disorder - consisting of random on-site energies for the outermost tight-binding sites, distributed between \pm 1 eV-the $G(L)$ traces become fairly insensitive to the value of $d$ (that is, chemical disorder or large structural disorder have identical effects; the data shown in the panel are obtained starting from an ideal zigzag edge). d, Further comparison of $G(L)$ traces calculated in the presence of strong disorder (in the universal limit) starting from armchair edges and for another crystallographic edge obtained by cutting the BLG edge at a $\theta \approx 10.9^{\circ}$ angle from the armchair direction (calculations were carried out with $d=20 a$ ). It is apparent that the results are virtually identical, confirming once again that for strong disorder different orientations become equivalent. The data shown in $\mathbf{d}$ have been calculated for samples of different widths $W$ (in units of number of atomic layers: circles correspond to $W=160$, squares to $W=200$, diamonds to $W=240$, triangles to $W=280$ ); the lack of sensitivity to $W$ indicates that subgap transport is dominated by edge states and not by the BLG bulk. The fact that transport is occurring at the edges can also be inferred from the data of a-c, which show that the conductance extrapolates in all cases to $G \rightarrow 2$ (in units of $2 e^{2} / h$ ) in the limit $L \rightarrow 0$ (corresponding to one spin-degenerate edge channel at each sample edge).

however, we find that for strong disorder the localization length tends to saturate to a rather large value, $\lambda^{*}$, that depends only on the magnitude of the gap (see Fig. 3 inset). The same behaviour is observed when starting from other periodic edges, obtained by cutting the BLG lattice along different crystallographic directions. The situation is different when starting from an ideal armchair edge, in which case we observe that both the conductance and localization length increase on increasing the disorder strength. The increase occurs because for an ideal armchair edge no states exist in the gap, and disorder introduces low-energy states at the edges that mediate transport. Remarkably, at large disorder strength the localization length also saturates to the value $\lambda^{*}$ found for disordered zigzag edges. When chemical disorder is also included (see Fig. $2 \mathrm{c}$ and points connected by the broken line in Fig. 3), the localization length saturates in all cases again to this same universal value, even for a very small amount of structural disorder $(d=1)$. Physically, this implies that in the presence of sufficiently strong disorder there is no relation between the structure of the starting (ideal) edge and the low-energy electronic properties. In other words, our calculations show that in the presence of sufficient disorder, be it structural or chemical, the electronic properties of graphene edges are universal, and they support low-energy electronic states responsible for subgap conduction.

The observation of a long and universal localization length is unexpected, and requires an explanation. To understand its origin we consider the evolution of the edge states with increasing disorder, and we focus on the behaviour of the enhanced penetration depth (that is, the depth by which edge states extend into the bulk). For perfect zigzag edges, the edge states at low energy decay into the bulk on the length scale $\ell_{0} \simeq a t / t_{\perp}$, with $t$ and $t_{\perp}$ being the intra- and inter-layer nearest-neighbour hopping energies, which is determined by the longitudinal momentum $k_{x}$ at which the energy of the edge states vanishes. In the presence of strong disorder, however, momentum is no longer a good quantum number, and the eigenfunctions of the low-energy states become superpositions of plane waves with all values of $k_{x}$. As a result the extent of the edge states away from the edge is determined by their momentum component that penetrates the most into the bulk. As can be easily seen from the analytic solution of the edge-state wavefunctions-confirmed by numerical calculations (see Supplementary Section SII) - the largest penetration depth is achieved for $k_{x}=0$, and is given by $\xi \simeq \ell_{0} / \sqrt{\Delta}>\ell_{0}$. This expression for $\xi$, which is parametrically larger than $\ell_{0}$, has the same functional dependence on $\Delta$ as the universal localization length $\lambda^{*}$. Indeed our numerical calculations confirm that $\lambda^{*}=C \xi$ with $C$ a constant prefactor found to be close to 5 . As $\xi$ is solely determined by the bulk properties of BLG, it is this proportionality between $\lambda^{*}$ and $\xi$ that explains the universality of $\lambda_{\text {loc }}$ at strong disorder. Physically, the fact that at strong disorder the localization length is determined by the penetration depth is intuitively clear: the deeper the electrons can 'escape' into the bulk, the less they will experience the disorder that is present at the edge. The proportionality between $\lambda^{*}$ and $\xi$ can be reproduced qualitatively by treating edge disorder within the selfconsistent Born approximation (see Supplementary Section SVIII). 


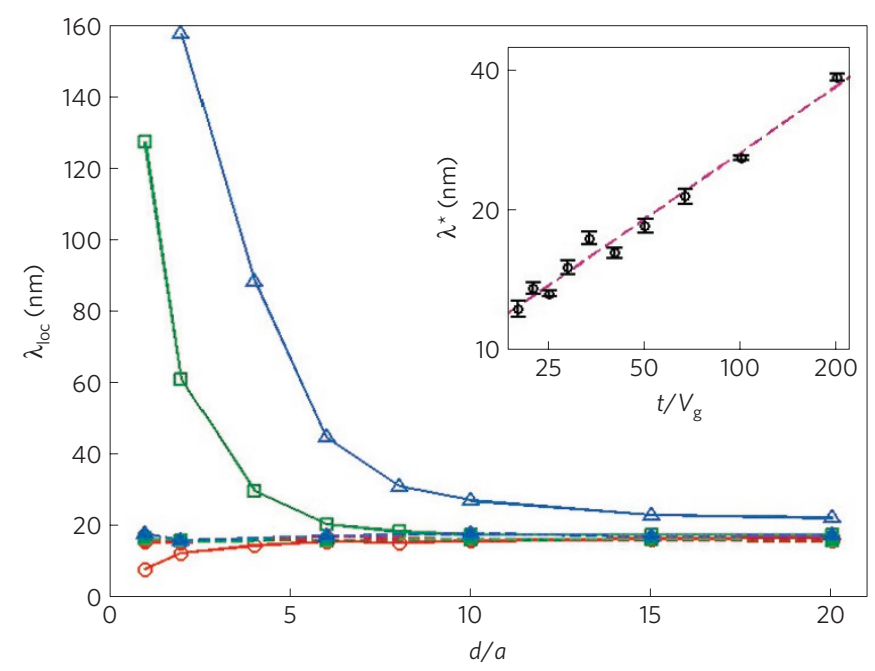

Figure 3 | Universal behaviour of localized edge states. The figure shows the dependence of the localization length $\lambda_{\text {loc }}$ on the amount of edge disorder, for the different orientations of the ideal 'starting' edges (the data are extracted from plots similar to those shown in Fig. 2: circles correspond to armchair edges, squares to a direction cut at an angle $\theta \approx 10.9^{\circ}$ from the armchair direction and triangles to zigzag edges). The cases in which only structural disorder is considered-quantified by the roughness depth $d$-correspond to the data points connected by solid lines. In these cases $\lambda_{\text {loc }}$ depends on both $d$ and $\theta$, until it saturates at large $d$. The cases in which strong 'chemical' disorder is also included, modelled by random on-site energies on the outermost row of carbon atoms, correspond to the data points connected by the broken line. With chemical disorder, the 'universal' value of the localization length is reached even for very small structural disorder (that is, $d \simeq 1$ ). In all cases, sufficiently strong disorder leads to the same value of $\lambda_{\text {loc }}$ regardless of any detail. This demonstrates that, for sufficiently strong disorder, the localization length of the edge states is universal, and depends only on the magnitude of the bandgap $V_{g}$. The universal localization length $\lambda^{*}$ for different values of the gap $V_{\mathrm{g}}$ is shown in the inset. We find that $\lambda^{*}$ is approximately proportional to $1 / \sqrt{V_{g}}$ (see the log-log plot in the inset, where the error bars indicate the $95 \%$ confidence intervals in fitting data similar to those shown in Fig. 2; the line is a fit yielding $\lambda^{*} \propto 1 / V_{g}{ }^{v}$ with $v=0.48 \pm 0.06$ ).

Finding that low-energy subgap edge states-with a very long localization length given the large strength of disorder consideredare present and characterized by universal properties is remarkable. To highlight the importance of the band-structure topology in the case of gapped BLG with disordered edges, we have carried out numerical calculations on a gapped half-filled square lattice (see Fig. 4a), which, similarly to BLG, is bipartite, that is, it is formed by two interpenetrating (square) sublattices. A gap in the electronic spectrum can be opened by unbalancing the sublattice energies (see Supplementary Information; we used values for the tight-binding hopping amplitude and for the gap comparable to those of graphene). Despite superficial similarity, unlike the case in BLG, the resulting band structure is topologically trivial. Accordingly, intragap states are neither expected nor observed numerically at the ideal edges. Indeed, we find that for the square lattice, transport in the ideal case is due to weak direct tunnelling under the gap. Edge roughness does not introduce edge states inside the gap, but only further suppresses conductance (see Fig. $4 \mathrm{~b}, \mathrm{c}$ ). This comparison underscores the fundamental importance of the topological origin of low-energy edge states in gapped BLG. Even though these edge states are not protected against short-range potential scattering, as is the case for spin-orbit-induced topological insulators $^{16,17}$, their robustness manifests itself in a very long and universal localization length.
Having established that the presence of low-energy edge states with long localization length is a generic property of gapped BLG, we discuss how these states manifest themselves in transport experiments. In this regard, the ability to tune the gap in BLG electrostatically up to values of $\approx 300 \mathrm{meV}$ (refs 22,23 ) is particularly useful. For large gap values, hopping via localized states is expected to be the dominant low-temperature transport mechanism. As the states localized at the edge naturally exist near the middle of the gap, they will give a dominant contribution to the measured conductance, as long as the potential fluctuations due to the bulk disorder are significantly smaller than the bulk gap ${ }^{24,25}$.

To compare with experiments, we estimate the characteristic energy scale that determines the temperature dependence of the conductance. To this end, we assume that transport is dominated by variable-range hopping along the edges (one-dimensional transport; the case of nearest-neighbour hopping would give a comparable result). The temperature dependence of the conductance can then be written in the form $G \propto \exp \left[-\left(T^{*} / T\right)^{1 / 2}\right]$, with $T^{*} \approx V_{\mathrm{g}} t_{\perp} / t$ (see Supplementary Information; here we have denoted the bandgap $2 \Delta$ by $V_{g}$, to emphasize that in the experiments this parameter can be controlled, and is proportional to the voltage applied between the gates). Therefore, $T^{*}$ is the characteristic energy scale that determines the low-temperature transport behaviour: like the bandgap, it increases with the applied gate voltage but it is approximately one order of magnitude smaller for the practically relevant values of $V_{\mathrm{g}}$. Indeed, in the first experimental observation of the insulating state in gapped BLG (ref. 26), the characteristic temperature scale extracted from the measurement was found to be more than one order of magnitude smaller than the gap (measured later by optical spectroscopy ${ }^{22,23}$ ). However, in the devices used in these first experiments, the BLG was in direct contact with the $\mathrm{SiO}_{2}$ gate insulator, which is known to cause sizable potential fluctuations ${ }^{24,25}$, and the relevance of the bulk contribution to the conductance cannot be excluded. Nevertheless, in recent experiments carried out on suspended, double-gated BLG (that is, with the bulk of the bilayer not affected by the direct contact with other materials) ${ }^{27}$, the maximum gap that could be opened electrostatically was estimated to be approximately $5 \mathrm{meV}$, whereas the resistance measured in d.c. transport exhibited an insulating behaviour with an activation energy of $0.3 \mathrm{meV}$. The difference between the expected bulk gap and the characteristic energy scale measured in transport is about one order of magnitude, consistent with our analysis. Given the cleanness of the suspended BLG samples, we argue that these experiments do indeed support the scenario presented here, namely that subgap transport is dominated by conductance at the edges of the graphene bilayer.

It is quite surprising that low-energy edge states in gapped BLG survive despite the presence of very strong disorder, even though the topological protection mechanisms, which operate in the spin-orbit-induced topological insulators, are absent. This finding illustrates the robustness of phenomena rooted in the underlying bulk band-structure topology, whose broad importance for condensed-matter physics is starting to be fully appreciated only now. A unique feature of BLG is that, by using gate electrodes to control the magnitude and sign of the gap between valence and conductance bands ${ }^{13}$, this underlying topology can be manipulated experimentally in nanoelectronic devices.

\section{Methods}

The rough edges of BLG samples in our simulations are generated by random walks (forward only) near the sample boundaries up to depth $d$, and 1,000 random configurations are averaged for each data point. The calculations are always done at the Fermi energy $E_{\mathrm{f}}=0$ for the samples, with highly doped contacts. The two-terminal conductance is calculated by using the recursive Green function method, and the local density of states is obtained from the spectral function. The important parameters for the BLG samples, unless otherwise specified, are $t=2.8 \mathrm{eV}, t_{\perp}=0.1 t, V_{\mathrm{g}}=0.03 t, a=1.42 \AA$ and $W=160$ (in units of number of atomic layers; interedge scattering is negligible with this width). 


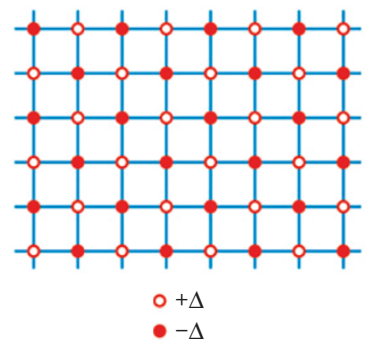

b

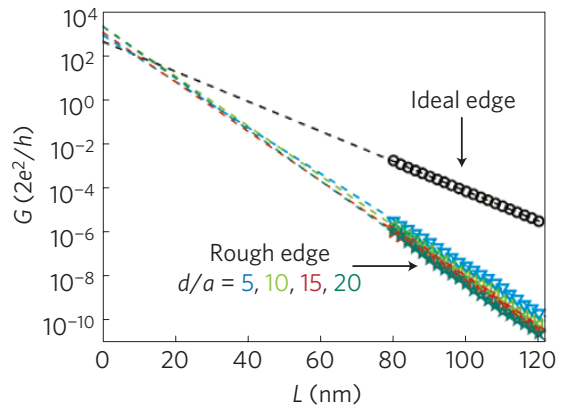

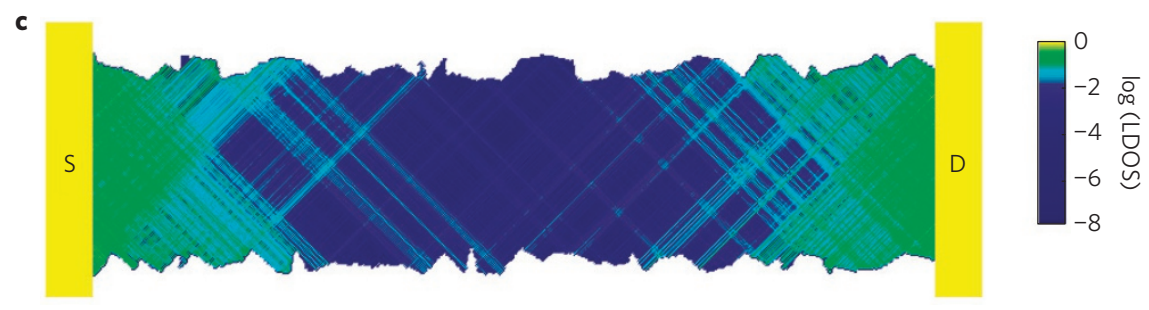

Figure 4 | Absence of disorder-induced edge transport in a topologically trivial system. a, As a term of comparison for gapped BLG, we analyse subgap transport in a square lattice, with a staggered potential $\Delta$ that determines the size of the gap, equal to $2 \Delta$ (the lattice constant $a$ is set to $a=1 \AA$ ). Subgap transport is investigated by setting the Fermi energy at the centre of the gap. $\mathbf{b}$, The subgap conductance $G$ as a function of strip length $L$, for the ideal edge, and for edges with different amounts of structural disorder. In this case edge roughness significantly lowers the conductance as compared with the case of ideal edges. In all cases, transport occurs through the bulk of the system and is due to evanescent modes decaying from the source and drain contacts (S and $D$ in $\mathbf{c}$ ), that is, it is due to tunnelling through the bulk gap. This is why, in contrast to gapped BLG, the conductance extrapolates to large values when $L \rightarrow 0$.c. The local density of states in a rough-edge sample $60 \mathrm{~nm}$ long. The scar-like trajectories in the bulk are caused by edge disorder, which affects the evanescent waves that decay exponentially away from the contacts. No evidence of edge states is found in this system.

\section{Received 26 March 2010; accepted 24 September 2010;} published online 21 November 2010

\section{References}

1. Son, Y., Cohen, M. L. \& Louie, S. G. Half-metallic graphene nanoribbons. Nature 444, 347-349 (2006).

2. Rycerz, A., Tworzydlo, J. \& Beenakker, C. W. J. Valley filter and valley valve in graphene. Nature Phys. 3, 172-175 (2007).

3. Guinea, F., Katsnelson, M. I. \& Geim, A. K. Energy gaps and a zero-field quantum Hall effect in graphene by strain engineering. Nature Phys. 6, 30-33 (2010).

4. Novoselov, K. S. et al. Two-dimensional gas of massless Dirac fermions in graphene. Nature 438, 197-200 (2005).

5. Zhang, Y., Tan, Y., Stormer, H. L. \& Kim, P. Experimental observation of the quantum Hall effect and Berry's phase in graphene. Nature 438, 201-204 (2005).

6. Novoselov, K. S. et al. Unconventional quantum Hall effect and Berry's phase of $2 \pi$ in bilayer graphene. Nature Phys. 2, 177-180 (2006).

7. Nakada, K., Fujita, M., Dresselhaus, G. \& Dresselhaus, M. S. Edge state in graphene ribbons: Nanometer size effect and edge shape dependence. Phys. Rev. B 54, 17954-17961 (1996).

8. Castro, E. V., Peres, N. M. R., dos Santos, J. M. B. L., Neto, A. H. C. \& Guinea, F. Localized states at zigzag edges of bilayer graphene. Phys. Rev. Lett. 100, 026802 (2008).

9. Yao, W., Yang, S. A. \& Niu, Q. Edge states in graphene: From gapped flat-band to gapless chiral modes. Phys. Rev. Lett. 102, 096801 (2009).

10. Brey, L. \& Fertig, H. A. Electronic states of graphene nanoribbons studied with the Dirac equation. Phys. Rev. B 73, 235411 (2006).

11. McCann, E. \& Fal'ko, V. I. Landau-level degeneracy and quantum Hall effect in a graphite bilayer. Phys. Rev. Lett. 96, 086805 (2006).

12. Volovik, G. The Universe in a Helium Droplet (Oxford Univ. Press, 2003).

13. Martin, I., Blanter, Y. M. \& Morpurgo, A. F. Topological confinement in bilayer graphene. Phys. Rev. Lett. 100, 036804 (2008).

14. Qi, X., Hughes, T. L. \& Zhang, S. Topological field theory of time-reversal invariant insulators. Phys. Rev. B 78, 195424 (2008).

15. Hasan, M. Z. \& Kane, C. L. Topological insulators. Rev. Mod. Phys. Preprint at http://arxiv.org/abs/1002.3895 (2010).

16. Bernevig, B. A., Hughes, T. L. \& Zhang, S. Quantum spin Hall effect and topological phase transition in HgTe quantum wells. Science 314, 1757-1761 (2006).

17. Kane, C. L. \& Mele, E. J. Quantum spin Hall effect in graphene. Phys. Rev. Lett. 95, 226801 (2005).
18. Kane, C. L. \& Mele, E. J. Z2 topological order and the quantum spin Hall effect. Phys. Rev. Lett. 95, 146802 (2005).

19. Konig, M. et al. Quantum spin Hall insulator state in $\mathrm{HgTe}$ quantum wells. Science 318, 766-770 (2007).

20. Roth, A. et al. Nonlocal transport in the quantum spin Hall state. Science 325, 294-297 (2009).

21. Mucciolo, E. R., Neto, A. H. C. \& Lewenkopf, C. H. Conductance quantization and transport gaps in disordered graphene nanoribbons. Phys. Rev. B 79, 075407 (2009).

22. Zhang, Y. et al. Direct observation of a widely tunable bandgap in bilayer graphene. Nature 459, 820-823 (2009).

23. Mak, K. F., Lui, C. H., Shan, J. \& Heinz, T. F. Observation of an electric-field-induced band gap in bilayer graphene by infrared spectroscopy. Phys. Rev. Lett. 102, 256405 (2009).

24. Martin, J. et al. Observation of electron-hole puddles in graphene using a scanning single-electron transistor. Nature Phys. 4, 144-148 (2008).

25. Kuzmenko, A. B., Crassee, I., van der Marel, D., Blake, P. \& Novoselov, K. S. Determination of the gate-tunable band gap and tight-binding parameters in bilayer graphene using infrared spectroscopy. Phys. Rev. B 80, 165406 (2009).

26. Oostinga, J. B., Heersche, H. B., Liu, X., Morpurgo, A. F. \& Vandersypen, L. M. K. Gate-induced insulating state in bilayer graphene devices. Nature Mater. 7, 151-157 (2008).

27. Weitz, R. T., Allen, M. T., Feldman, B. E., Martin, J. \& Yacoby, A. Coulomb-driven broken-symmetry states in doubly gated suspended bilayer graphene. Preprint at http://arxiv.org/abs/1010.0989 (2010).

\section{Acknowledgements}

This work has been supported by the Swiss National Science Foundation (projects 200020-121807 and 200021-121569) and by the Swiss Centre of Excellence MaNEP. The work of I.M. was carried out under the auspices of the National Nuclear Security Administration of the US Department of Energy at Los Alamos National Laboratory under contract No. DE-AC52-06NA25396 and supported by the LANL/LDRD Program.

\section{Author contributions}

All authors contributed to the discussion of the ideas presented in the paper, and to the analytical calculations. J.L. carried out all the numerical computations.

\section{Additional information}

The authors declare no competing financial interests. Supplementary information accompanies this paper on www.nature.com/naturephysics. Reprints and permissions information is available online at http://npg.nature.com/reprintsandpermissions. Correspondence and requests for materials should be addressed to A.F.M. 